

\title{
Evolution of microstructure and twin density during thermomechanical processing in a $\gamma-\gamma^{\prime}$ nickel-based superalloy
}

\author{
Nathalie Bozzolo, Nadia Souaï, Roland E. Logé
}

\section{- To cite this version:}

Nathalie Bozzolo, Nadia Souaï, Roland E. Logé. Evolution of microstructure and twin density during thermomechanical processing in a $\gamma-\gamma^{\prime}$ nickel-based superalloy. Acta Materialia, 2012, 60 (13), pp.50565066. 10.1016/j.actamat.2012.06.028 . hal-00722027

\section{HAL Id: hal-00722027}

https: / hal-mines-paristech.archives-ouvertes.fr/hal-00722027

Submitted on 22 Aug 2012

HAL is a multi-disciplinary open access archive for the deposit and dissemination of scientific research documents, whether they are published or not. The documents may come from teaching and research institutions in France or abroad, or from public or private research centers.
L'archive ouverte pluridisciplinaire HAL, est destinée au dépôt et à la diffusion de documents scientifiques de niveau recherche, publiés ou non, émanant des établissements d'enseignement et de recherche français ou étrangers, des laboratoires publics ou privés. 


\title{
Evolution of microstructure and twin density during thermomechanical processing in a $Y-\gamma^{\prime}$ nickel-based superalloy
}

\author{
N. Bozzolo*, N. Souaï, R.E. Logé
}

MINES ParisTech, CEMEF - Centre de Mise en Forme des Matériaux, CNRS UMR 7635, BP 2071 rue Claude Daunesse 06904 Sophia Antipolis cedex, France

E-mail adresses:

nathalie.bozzolo@mines-paristech.fr, nadia.souai@mines-paristech.fr, roland.loge@minesparistech.fr

${ }^{*}$ Corresponding author:

Nathalie Bozzolo, phone : +33 4936789 45, fax: +33 492389752

\section{Abstract.}

Microstructure evolution has been studied in the nickel-based superalloy PERß72 submitted to hot-torsion, to annealing below the primary $\mathrm{Y}^{\prime}$ solvus temperature and to annealing at a supersolvus temperature, with a special emphasis on grain size and twin content. Dynamic abnormal grain growth occurs before the onset of dynamic recrystallization. The resulting bimodal grain size distribution affects the grain coarsening kinetics at the supersolvus temperature, so that the final microstructures depend on the former straining stages. As a consequence, the twin content does not follow a univocal relationship with the average grain size. The grain boundary velocity history before reaching the final grain size is a contributing factor, and this is notably related to the initial grain size distribution width. Dynamically recrystallized microstructures are by nature more homogeneous and give then rise to lower rates in supersolvus grain coarsening, and accordingly lead to relatively lower twin densities.

\section{Keywords.}

Ni-based superalloys, Thermomechanical processing, Microstructure, Twin boundary, Grain boundary engineering 


\section{Introduction / Background}

Nickel-based superalloys have been specifically designed for high temperature applications and are mostly used in aeronautical components. Besides the development of new alloy compositions, efforts have been made to control the microstructure evolution during thermomechanical processing of existing alloys. Grain size and second phase topology are well-known key factors to get the required properties in $\gamma-\gamma^{\prime}$ nickel-based superalloys such as Udimet 720 [1-6]. The grain boundary network optimization is another possible route [7-12]. The principle of Grain Boundary Engineering (GBE) consists in tailoring the Grain Boundary Character Distribution (GBCD) to improve some of the material properties, especially those related to intergranular damage [13]. It has been successfully applied on a number of low stacking fault energy metals and alloys. The beneficial boundaries, so-called special boundaries, are usually based on $\Sigma 3$ twin boundaries and other related low $\Sigma$ boundaries [14]. Most of the related papers address either the influence of "special boundaries" on properties or the control of the fraction of those "special boundaries" by thermomechanical processing, but very few is known about the fundamental metallurgical mechanisms controlling the special boundary genesis. It is worth noticing that $\Sigma 3$ twin boundaries themselves can sometimes be detrimental for specific properties [15] and then should on the contrary be avoided. Beneficial or detrimental, the control of the $\Sigma 3$ twin density appears to be necessary.

For materials like nickel-based superalloys usually submitted to fatigue and creep-fatigue conditions in aeronautical components, grain boundary engineering seems particularly attractive. One of the difficulties to be overcome is that most of the developed grain boundary engineering routes are based on cycling cold deformation and annealing stages; nickelbased superalloys have to be processed at high temperature. In addition second phase particles are often mandatory to avoid grain coarsening during thermomechanical processing; the materials on which grain boundary engineering has been applied were mostly single phase.

In a previous paper [16], variations in $\Sigma 3$ twin content as a function of thermomechanical parameters have been shown in PER®72 microstructures that were supersolvus-annealed after deformation. In addition twin amounts were not fully dependent on grain size, which is a conclusion that supports the possibility of developing grain boundary processing routes involving hot deformation. The results of this previous work allowed formulating hypotheses concerning the involved mechanisms, which are investigated in more details in the present paper, by inspecting microstructure at intermediate stages: deformed and quenched, and 
also after subsolvus annealing. The overall microstructure evolution mechanisms will be discussed, with a special emphasis on twin genesis and grain size evolution.

\section{Material and experiments}

\subsection{Material}

The studied material is the two phase $\left(\gamma-\gamma^{\prime}\right)$ nickel-based superalloy PER®72 (equivalent to the alloy $720 \mathrm{LI}$ ), provided by Aubert\&Duval in the form of a forged bar, which is the usual state of the material before disc forging. The microstructure of the as-received state is composed of fine $(\sim 7 \mu \mathrm{m})$ equiaxed grains of the $\mathrm{y}$ phase (FCC solid solution) and of $\gamma^{\prime}$ precipitates $\left(\mathrm{Ni}_{3}(\mathrm{Al}, \mathrm{Ti})\right.$, ordered $\mathrm{L} 1_{2}$ cubic structure). With a typical size of 1-3 $\mu \mathrm{m}$ and a rounded shape, the latter are primary precipitates which formed during the alloy solidification. Secondary and tertiary ones $(<0.5 \mu \mathrm{m})$ also form from the supersaturated solid solution state during cooling and aging treatment.

\subsection{Thermomechanical processing}

Torsion test samples have been machined from the bar in order to perform hot-deformation at a constant deformation rate $\left(10^{-1} \mathrm{~s}^{-1}\right)$ and a subsolvus temperature $\mathrm{T}_{\text {sub. At }}$. this temperature, the primary $Y^{\prime}$ precipitates do not dissolve, or at least do not completely dissolve, but the secondary and tertiary ones do. Samples were deformed up to different strain levels and then immediately water quenched to freeze the deformed microstructure. For studying the microstructure evolution during static annealing, samples were subsequently heated back to the subsolvus temperature or to a higher supersolvus temperature $\left(T_{\text {sup }}\right.$, where the $\mathrm{Y}^{\prime}$ phase completely disappears). A constant annealing time of 30' was applied. The performed thermomechanical treatments are summarized in Fig. 1. For each applied strain $(\varepsilon=0.12$, 0.40 or 0.63 ), three microstructures were then produced: i) deformed-and-quenched, ii) deformed-and-quenched and further annealed for $30^{\prime}$ at $\mathrm{T}_{\text {sub }}$, and iii) deformed-and-quenched and further annealed for 30 ' at $\mathrm{T}_{\text {sup. }}$. Consistently, the initial material was also characterized i) after a 10' annealing at $\mathrm{T}_{\text {sub }}$ (for the sake of comparison with the deformed-and quenched samples, which were held for $10^{\prime}$ at $\mathrm{T}_{\text {sub }}$ while performing the torsion test), ii) after annealing for $30^{\prime}$ at $T_{\text {sub }}$, and iii) after annealing for $30^{\prime}$ at $T_{\text {sup. }}$.

\subsection{Microstructure characterization methods}

\subsubsection{Orientation mapping}


The EBSD technique was used for microstructure characterization because it allows obtaining quantitative data on the grain structure as well as on the nature of the grain boundaries. Sample preparation involved classical polishing on SiC papers, then with $3 \mu \mathrm{m}$ and $1 \mu \mathrm{m}$ diamond powder, and finally on a vibrating plate during $15 \mathrm{~h}$ using $0.05 \mu \mathrm{m}$ alumina particles. The EBSD system is implemented in a FEI XL30 scanning electron microscope equipped with $\mathrm{L} \mathrm{LaB}_{6}$ cathode. Under $20 \mathrm{kV}$ acceleration voltage, the ultimate spatial resolution achievable in the orientation maps was around $300 \mathrm{~nm}$. The samples deformed and annealed at $T_{\text {sub }}$ had a rather small grain size; the chosen step size was $400 \mathrm{~nm}$. For the ones annealed at $T_{\text {sup }}$, grains were much larger, and the step size could be increased to 1.5 $\mu \mathrm{m}$, which still provided a reasonable microstructure detail resolution. Large maps including at least 1000 y-grains were acquired in order to ensure the statistical relevance of the measured microstructural parameters. Only small pieces of those maps are shown here.

In practice, the EBSD indexing software was unable to distinguish $y$ and $\gamma^{\prime}$ phases, because first their crystallographic structures are very similar and second the $y$-matrix includes many coherent secondary $\gamma^{\prime}$ precipitates at room temperature. The $y$ matrix is face-centred-cubic (FCC). The $Y^{\prime}$ precipitates have a simple cubic structure resulting from atom ordering on a FCC lattice with a cell parameter misfit of only about $1 \%$ with the matrix. The phase separation has therefore been done a posteriori using a double criterion based on grain size and on volume fraction. The complete post-processing procedure that was designed to analyze the EBSD maps includes four steps described below, and was applied only to reliable data, i.e. not considering pixels with a confidence index (TSL-OIM software parameter ranging from 0 to 1 ) lower than 0.15 .

\section{1) Grain detection}

The isolated non-indexed pixels and the isolated pixels with an orientation different from the neighbourhood are preliminarily corrected. Then grains are detected as groups of adjacent pixels either with less than $10^{\circ}$ disorientation or a misorientation close to the $\Sigma 3$ twin orientation relationship. The twin boundaries were therefore ignored in the grain detection procedure.

2) Partitioning of $y$ and $y^{\prime}$ phases

The $Y^{\prime}$ precipitates are smaller than the $Y$ grains. Typically the largest precipitates do not exceed $3 \mu \mathrm{m}$ in diameter, as it can be seen on the BSE micrograph of Fig. 2 where the rounded shape $\gamma^{\prime}$ precipitates appear somewhat darker than the matrix. In addition, the overall chemical composition drives the thermodynamic equilibrium $\gamma^{\prime}$ volume fraction to about $18 \%$ at $\mathrm{T}_{\text {sub }}$ (value given by the alloy supplier). The smallest grains accounting for $18 \%$ 
of the map area have therefore been considered to mainly be $y^{\prime}$ precipitates and were then not considered in further $y$-phase characterizations.

3) y-grain size

The equivalent circle diameter was calculated for each $y$-grain, so that both a grain size histogram could be built and an average grain size $\bar{D}$ computed:

$\bar{D}=\frac{\sum_{i} A_{i} \cdot D_{i}}{\sum_{i} A_{i}}$

where $A_{i}$ and $D_{i}$ are the area and the equivalent circle diameter of the $i^{\text {th }}$ grain, respectively. If the $2 \mathrm{D}$ measured distribution of grain sizes must be converted to a $3 \mathrm{D}$ distribution for a modelling purpose, the classical Saltykov method is used [17].

\section{4) $\Sigma 3$ twin boundary quantification}

Twin boundaries quantification has been performed for the $\mathrm{y}$ phase only, using a program specifically developed to analyse the filtered EBSD maps. Only $\Sigma 3$ twins have been analyzed because the amount of higher order coincidence-site-lattice (CSL) boundaries, including second and third generation twins, were very small and their variations were therefore not significant. A tolerance of $2^{\circ}$ or $4^{\circ}$ disorientation with respect to the theoretical $60^{\circ}<111>$ misorientation was used in $\Sigma 3$ twin boundaries detection. The amount of $\Sigma 3$ twin boundaries is quantified as being the length of the considered type of boundary per unit area and referred to as $d_{\Sigma 3}^{2^{\circ}}$ or $d_{\Sigma 3}^{4^{\circ}}\left(\mu m^{-1}\right)$. Furthermore, coherent and incoherent twins were discriminated: the trace of the plane of a coherent twin boundary had to be disoriented by less than $5^{\circ}$ relatively to the closest $\{111\}$ plane trace; otherwise the twin was classified as incoherent. The length per unit area has been used as a quantification parameter for $\Sigma 3$ twin boundaries, regardless of the coherent/incoherent character, and the length fraction of incoherent $\Sigma 3$ twin boundaries has additionally been calculated (referred to as \%incoh.). For incoherent twin discrimination, the misorientation tolerance angle was set to $4^{\circ}$.

\section{Microstructure ( $\gamma$ matrix grain structure) evolution mechanisms}

In the following sections, EBSD results obtained on all samples will be described. Two EBSDmap representation modes will be used to show the grain structure of the $y$ matrix and the corresponding grain boundary network. The grain structure plot consists in a random 
colouring of $y$ grains, the small grains filtered as $Y^{\prime}$ precipitates being left white. On the other hand, the grain boundary network is displayed by drawing a red line for $\Sigma 3$ twin boundaries, and a black one for any other grain boundary, including $Y-\gamma^{\prime}$ interfaces.

\subsection{Deformed-and-quenched materials}

The stress-strain curve obtained from a torsion test (Fig. 3) exhibits a pronounced stress drop at the very beginning of the plastic domain (at $\varepsilon=0.01-0.02$ ) immediately followed by a maximum at $\varepsilon=0.03$. Similar yield point phenomena have been reported in other nickelbased superalloys [18]. The locking mechanism was then identified as the interaction of dislocations with alloying elements which may undergo short-range ordering or precipitation. After this, the strain-stress curve adopts a more classical aspect, with a slight hardening up to $\varepsilon=0.1-0.15$ followed by a slow softening. At $\varepsilon=0.1-0.15$ the plastic flow stress reaches a maximum value that is $8-10 \%$ higher than the subsequent plateau value. The flow stress is then progressively decreasing to a reach a steady state value of $106 \mathrm{MPa}$ at $\varepsilon=0.35-0.40$. Such a decrease in flow stress is usually attributed to the activation of softening mechanisms like dynamic recovery and recrystallization. Torsion tests have been interrupted at $\varepsilon=0.12$, 0.40 or 0.63 to observe the microstructure of the most hardened state (stress peak), the fullysoftened state (onset of the plateau), and the steady state (further on the plateau), respectively.

The grain structures obtained after deformation and quenching (Fig. 4) are almost equiaxed whatever the applied strain, as the case for the undeformed reference material. The unchanged grain shape suggests the occurrence of dynamic grain boundary motion. Superplastic grain boundary sliding which is the other possible mechanism is indeed very unlikely under the applied conditions of strain rate, temperature and initial grain size.

Contrary to the grain shape, the grain size and the width of the grain size distribution are dependent on the applied strain. The reference material is homogeneous with a rather small grain size $(\bar{D}=7.5 \mu \mathrm{m})$. After 0.12 straining, the material is on the contrary heterogeneous, with a bi-modal grain size distribution leading to a $\bar{D}$ value of $15.0 \mu \mathrm{m}$. Dynamic grain coarsening obviously occurred selectively for few grains at such low strains. Observing the microstructure over a wider area and with a better spatial resolution, it appears that the big grains form bands in which the precipitates density is lower (Fig 5a). The heterogeneity in the spatial distribution of the primary $Y^{\prime}$ precipitates is directly linked with chemical segregations which can not be avoided in such heavily alloyed materials. The lower the precipitate density, the lower the grain boundary pinning force. One could therefore think that the development of 
the big grains is a direct consequence of the Zener pinning force being insufficient in those areas to prevent grain boundary motion. But if so, large grains should also appear in the initial material submitted to the $10^{\prime}$ heat treatment at $\mathrm{T}_{\text {sub }}$, which has similar chemical segregations. This does not happen (Fig. 5b). The material which has been deformed has an additional driving force which allows overcoming the Zener pinning force in selected zones. Strain Induced Grain Boundary Motion (SIBM) is a reasonable explanation for that phenomenon [19, 20]. Depending on their crystallographic orientation, grains store different dislocation densities. Gradients of dislocation densities across grain boundaries create a driving force for their motion, and favour the dynamic growth of the grains with the lowest energy level. In the present alloy, the local development of large grains in the slightly deformed sample is due to the combination of two conditions: lower precipitate density due to chemical segregation, and additional driving force for grain boundary motion induced by strain energy density fluctuations.

If the material is further deformed at $\varepsilon=0.40$, the microstructure becomes homogeneous again, with a grain size in the same range as for the reference sample $(\bar{D}=9.0 \mu \mathrm{m})$. Dynamic recrystallization is then likely to be responsible for both the stress decrease between $\varepsilon=0.12$ and $\varepsilon=0.40$ and the disappearance of the previously formed large-grain

population. The steady state grain size corresponding to the flow stress plateau $(\bar{D}=16.6$ $\mu \mathrm{m}$ measured after $\varepsilon=0.63$ ) is somewhat larger and remains in the same range after larger strains (not shown here). This suggests that, in the two-phase material, reaching the microstructural steady state requires higher strains than reaching the flow stress steady state.

\subsection{Deformed, quenched and subsolvus annealed materials}

The evolution of the previous microstructures during a subsequent annealing for 30 ' at $\mathrm{T}_{\text {sub }}$ is presented on Fig. 6 . The undeformed material undergoes a uniform grain coarsening, almost by a factor of 2 , to reach a mean grain size of $13.3 \mu \mathrm{m}$, the grain boundary motion driving force was therefore high enough in the reference material to counterbalance the pinning force exerted by the primary- $\gamma^{\prime}$ precipitates. For the deformed samples, the $T_{\text {sub }}$ annealing reinforces the microstructure features observed after deformation. In the sample that was deformed to $\varepsilon=0.12$, the abnormally grown grains become even larger, which results in an increase in the mean grain size. The microstructure obtained after deformation to $\varepsilon=0.40$ (supposed to be fully dynamically recrystallized) keeps the same mean grain size $(\bar{D}=9.0$ $\mu \mathrm{m}$ ) after $\mathrm{T}_{\text {sub }}$ annealing, but the grain size distribution is somewhat broader. Both the largest grains (sizes above $16 \mu \mathrm{m}$ ) and the smallest ones (sizes below $6 \mu \mathrm{m}$ ) increase in area 
fraction, at the expense of intermediate grain sizes. This is not a classical behaviour, during conventional grain coarsening the largest grains develop at the expense of the smallest ones. Here in the dynamically recrystallized microstructure, the smallest grains are likely to be those which recrystallized the latest, and with therefore lower stored energy levels, and the potential to grow despite their small size. Grains of intermediate sizes have on the contrary higher stored energy levels because they appeared sooner in the microstructure and have subsequently been strain hardened. This explains why they are preferentially consumed by the growing grains. While being consumed, they become smaller and smaller and contribute to the increase of the smallest grain population. Some of the largest grains on the other hand may keep growing due to their size advantage. In the steady state material $(\varepsilon=0.63), \mathrm{T}_{\text {sub }}$ annealing only promotes a very slight increase in the mean grain size as compared to the deformed and quenched sample (18.3 vs $16.6 \mu \mathrm{m})$. The area fraction of large grains has increased, and that of the smallest grains has on the contrary decreased. As compared to the previous case, the stored energy distribution and its correlation to the grain size may have changed so that the evolution of the grain size distribution is more conventional.

In all three microstructures, the grain size distributions have slightly changed during the $T_{\text {sub }}$ annealing, showing that the pinning forces exerted by the precipitates did not completely prevent the boundaries from moving, at least locally.

\subsection{Deformed, quenched and supersolvus annealed materials}

The evolution of the deformed and quenched microstructures during a subsequent annealing at a supersolvus temperature, where the primary $V^{\prime}$ precipitates completely dissolve, is presented on Figs. 7 and 8. Grain coarsening is much easier at $T_{\text {sup }}$ than at $T_{\text {sub }}$ because the precipitate pinning force vanishes and because temperature is higher. Significantly larger grain sizes are then reached. Grains are equiaxed and with rather homogeneous size distributions. The most striking result of this series is that the grain coarsening amplitude still depends on the previous $T_{\text {sub }}$ deformation. This is obvious when comparing the sample deformed to $\varepsilon=0.12$ and the one deformed to $\varepsilon=0.63$, which had almost the same mean grain size in the deformed-and-quenched state $(15.0$ and $16.6 \mu \mathrm{m})$, and reach after supersolvus annealing a mean grain size of 90.3 and $50.3 \mu \mathrm{m}$ respectively.

The supersolvus grain coarsening amplitude is similar in the two samples deformed to strains corresponding to the flow stress steady state $(\varepsilon=0.40$ or 0.63$)$. For the samples deformed to lower strains ( $\varepsilon=0.08,0.12$ or 0.24 , shown on Fig. 8 ), the factor by which the grain size is increased during the supersolvus annealing is significantly greater, and is decreasing with 
increasing strain. Those materials were not at all or partially dynamically recrystallized during the torsion test. The stored energy level was therefore higher in these samples. The contribution of the stored energy to the driving force for microstructure evolution can nevertheless hardly explain the faster grain coarsening in the low strained microstructures. Stored energy is indeed very likely to be released fast at the supersolvus temperature (due to recovery and grain boundary migration), and even probably already disappeared while heating. Another feature of those samples is the bi-modal grain size distribution, and this is one cause of the larger increase in the mean grain size during supersolvus annealing. The grain coarsening is driven by the grain boundary curvature. Large grains in a matrix of small grains have a higher number of neighbors, and therefore have concave grain boundaries with a high curvature. The velocity of the boundaries can be expressed as the product of the mobility and the curvature induced pressure, which can be expressed as [21, 22] :

$$
P \approx \gamma\left(\frac{1}{\left.<R_{i}\right\rangle}-\frac{1}{R_{i}}\right)
$$

where $\mathrm{Y}$ stands for the grain boundary energy and $\mathrm{R}_{\mathrm{i}}$ for the "equivalent" grain radius. The two grain size distributions obtained after straining to $\varepsilon=0.12$ and 0.63 , and quenching (Fig. 4), have been converted to 3D distributions using the Saltykov method, and considered as "initial" microstructures for the simulation of grain growth during a supersolvus heat treatment. Neglecting stored energies and applying equation (2) to those distributions leads to final 3D distributions, which are given in Fig. 9. These are compared to the experimental data of Fig. 7, also converted to 3D distributions. Due to the arbitrary choice of the mobility and grain boundary energy parameters, the time of the simulation was adjusted such that the simulated grain growth after $\varepsilon=0.63$ led to the same (volume) average $3 \mathrm{D}$ grain size as the experimental one, i.e. $58.4 \mu \mathrm{m}$. With this simulated time, the obtained average grain size after $\varepsilon=0.12$ was $78.1 \mu \mathrm{m}$, against $102.8 \mu \mathrm{m}$ experimentally. The $20 \mu \mathrm{m}$ increase in the average grain size is a direct consequence of the shape of the initial distribution being wider in the latter " $\varepsilon=0.12$ " case as compared to the previous " $\varepsilon=0.63$ " one.

Grain growth simulations assuming homogeneous microstructures and no precipitate pinning effects could nevertheless not capture the distribution upper tails measured experimentally (Fig. 9). The tail was more pronounced in the sample pre-strained at $\varepsilon=0.12$, which explains why the predicted average grain size remained below the experimental value. The origin of such upper tails may be attributed to the inhomogeneous precipitate density (Fig. 5a): in the zones with lower density, Zener pinning forces are lower, leading to earlier grain growth upon heating. It is expected that larger pre-strains lead to a change in the spatial arrangement of precipitates which tend to homogenize pinning forces. 
The width of the grain size distribution therefore appears to be an important factor in the control of the grain growth, but also second phase particle local depletion which favours the development of the upper tail in the grain size distribution. Both effects are consistent with the grain coarsening amplitude decreasing with increasing strain, because the microstructure homogenizes as strain increases and as dynamic recrystallization progresses.

\section{Quantitative $\Sigma 3$ boundaries analysis}

The evolution of the $\Sigma 3$ boundary density and of their coherent/incoherent character is presented in Fig.10 a-c.

In the quenched materials, the $\Sigma 3$ boundary density seems to increase very slightly at low strains (before the maximum stress peak), but afterwards it decreases with increasing strain (Fig.10a). After subsolvus annealing, the $\Sigma 3$ boundary density is systematically higher than in the corresponding quenched samples. The microstructural re-organization events that occur during the subsolvus annealing obviously promote twin formation. On the contrary, if the annealing is performed above the supersolvus temperature, the $\Sigma 3$ boundary density drastically decreases, by a factor of more than 2 .

Annealing twins form while grain boundaries are moving [23, 24]. According to Pande et al. [25], the number of twins produced is proportional to the distance over which grain boundary have moved (related to the increase in grain size), and to the velocity of the grain boundary. Based on theses considerations, they proposed an analytical model for prediction of the twin density resulting from curvature driven grain growth, which fit very well with a large number of experimental results reported for various metals (Fig.11). The general trend is a decrease in twin density as grain size increases, because the velocity of the grain boundary is decreasing as grain size and grain boundary curvature decrease. For small grain sizes the trend is inverted, because of the migration distance which is then too small to promote twin formation. It is worth mentioning that the twin density is quantified as a number of twin intercepts per unit length. Our definition of twin density is somewhat different, defined as the length of twin segments per unit area. Based on general stereology concepts [26], both quantities are related by a proportionality factor. The conversion is therefore easy: the length per unit area equals the number of intercepts per unit length multiplied by $\pi / 2$, but it has not been done here since only relative changes in twin density will be discussed below.

In our results, the twin density remains constant, or even slightly increases, as grain size is increased by a factor of 3 in the samples submitted to curvature driven grain growth during supersolvus annealing (square symbols in Fig.10b). For such an increase in grain size, 
Pande's model predicts that the twin density should be divided by two. In Pande's approach, the curvature driving force is simply considered to be proportional to $\frac{Y}{<R>}$, and the effect of the grain size distribution width is neglected. It was shown in the previous section, however, that this effect cannot be neglected during supersolvus annealing. The largest grain sizes are obtained from the microstructures in which a bimodal grain size distribution has been produced during the former hot-deformation stage. Accordingly, the grain boundary migration rate during supersolvus annealing is increased, and the resulting twin density is significantly higher than that predicted by Pande's model.

The grain boundary velocity is also likely to be responsible for the increase in twin content during subsolvus annealing. In presence of the primary $V^{\prime}$ particles, the grain size does not change much, but grain boundaries may locally reorganize (in between pinning points) under stored energy gradient induced pressures. This would occur over short distances but at a high velocity, and would therefore promote the formation of twins. Another hypothesis is a reorganization of the twin boundaries themselves during the subsolvus annealing [27]. The length fraction of incoherent segments among all $\Sigma 3$ twin boundaries is shown in Fig. 10c as a function of strain. It is worth noticing that these results are rather scattered, the discrimination between coherent and incoherent twins would have been more accurate with higher spatial resolution maps. Trends can nevertheless be discussed. Low strain samples (in which the dynamic recrystallization onset was not reached) show the highest proportion of incoherent twins in the quenched state and a somewhat lower proportion after subsolvus annealing. On the contrary, supersolvus microstructures show rather few incoherent twins, which suggests that the twins formed during grain growth are mainly coherent ones. Fig.10d allows evaluating how far twin boundaries are from the theoretical $\Sigma 3$ orientation relationship (independently of their coherent/incoherent character) : it shows the percentage of twin boundaries detected within a $2^{\circ}$ tolerance among the ones detected with a $4^{\circ}$ tolerance. Here again the twins of the supersolvus annealed samples appear to be the most perfect ones, whereas the ones in the quenched samples may deviate more from the theoretical orientation relationship. Fig.12 shows a higher resolution EBSD map of a sample quenched after a deformation of $\varepsilon=0.24$, which indeed reveals that deformation induced crystallographic orientations changes and the interaction between intragranular dislocation structures (subboundaries) and twin boundaries may both result in deviating from the $\{111\}$ plane (single arrow), or getting out of the angular tolerance for twin detection (double arrow). The preexisting twins can therefore be partly destroyed as a result of plastic deformation, but defects 
may annihilate during the subsequent subsolvus annealing, and boundaries may reorient [27], leading to the possible recovery of perfect coherent twins.

The above suggested mechanisms are still hypothetical; further investigations are needed to better understand how twins evolve during plastic deformation and subsequent annealing. Another important aspect which is still to be studied is the influence of the second phase particles on twin formation probability. Figure 13 shows $\Sigma 3$ twin boundaries, and grain and interphase boundaries in a microstructure strained to $\varepsilon=0.12$ and subsequently annealed at the subsolvus temperature. Primary $\gamma^{\prime}$ particles can be recognized, especially the intragranular ones, thanks to their rounded shape. Many twins terminate on second phase particles, suggesting that particles may play a role in the twin formation mechanisms. Second phase particles are well known to slow down grain boundary motion, and this would according to the above developed considerations based on Pande's approach - decrease twin density. When considered at the local scale, though, the interaction of a particle with a moving grain boundary can be on the contrary considered as an accelerating factor. If the driving force is high enough so that the grain boundary can be unpinned from the particle, at that very moment, the local velocity can be very fast, and promote twin nucleation. The presence of second phase particles would then increase the probability of twinning. This is an important fundamental issue which calls for further investigation.

The effect of prior deformation is not taken into account either in Pande's model, but this could be done in a more physically based model originally proposed by Gleiter [23], and further refined and validated by Li et al. [28]. In the latter, twin formation is treated as a nucleation event, and the probability of twin formation derived according to the classical thermodynamic description of nucleation events. This model could easily account for stored energy effects because the difference in free enthalpy between neighboring grains explicitly appears. However Gleiter's model requires the determination of many physical quantities which are not always known. In this paper, we have shown that two other parameters may be of prime importance in the control of the grain boundary velocity, and therefore in the resulting twin density: the grain size distribution width, and the presence of second phase particles. The existing models predicting twin densities should be extended in order to account for such effects. 


\section{Conclusions}

From examination of torsion test samples of the PER®72 alloy, in the quenched state, or annealed state at a temperature below or above the dissolution temperature of primary $Y^{\prime}$ particles, it appeared that:

(1) Dynamic abnormal grain growth occurs despite primary $\gamma^{\prime}$ particles, by strain induced grain boundary motion. Low strains and lower particles densities favour the development of those large grains. They disappear subsequently with dynamic recrystallization.

(2) The resulting bi-modal grain size distribution leads to faster grain coarsening during supersolvus annealing. This is one of the reasons for which the microstructures obtained after supersolvus annealing are still dependent on the thermomechanical history.

(3) The grain boundary velocity is an important factor in controlling twin density. Going through bi-modal grain size distributions allows reaching large grain sizes at a higher rate, and therefore with increased twin content, as compared to curvature driven grain growth in microstructures characterized by single-peak and narrow distributions. The twin density is then not univocally linked to the grain size.

(4) Controlling the final twin content in a two-phase nickel-based superalloy requires to consider the effects of i) stored energy gradients, ii) second phase particles, and iii) grain size distribution width, on grain boundary motion. None of the models reported in the literature can account for these three effects.

\section{Acknowledgements}

This work has been funded by the French Research National Agency (ANR project called Organdi and involving Safran-Snecma, Safran-Turbomeca, Eramet-Aubert\&Duval, CEA, ONERA, ENSMA and MINES-ParisTech). The authors would like to thank MSR staff members (G. Fiorucci and S. Jacomet) for their help in carrying out hot-torsion tests and EBSD analyses, analyses, and M. Bernacki and O.Beltran for their help in running the 3D grain growth simulations.

\section{References}

[1] F. Torster, G. Baumeister, J. Albrecht, G. Lütjering, D. Helm, M.A. Daeubler "Influence of grain size and heat treatment on the microstructure and mechanical properties of the nickel-base superalloy U 720 LI", Materials Science and Engineering: A 234-236 (1997) 189-192 
[2] M.P. Jackson, R.C. Reed "Heat treatment of UDIMET 720Li: the effect of microstructure on properties", Materials Science and Engineering: A 259 (1999) 85-97

[3] H. Monajati, M. Jahazi, R. Bahrami, S. Yue "The influence of heat treatment conditions on $Y^{\prime}$ characteristics in Udimet $\AA$ 720" Materials Science and Engineering: A 373:1-2 (2004) 286-293

[4] R. Radis, M. Schaffer, M. Albu, G. Kothleitner, P. Pölt, E. Kozeschnik "Multimodal size distributions of $\mathrm{Y}^{\prime}$ precipitates during continuous cooling of UDIMET 720 Li" Acta Materialia, 57:19 (2009) 5739-5747

[5] E. Chateau, L. Rémy "Oxidation-assisted creep damage in a wrought nickel-based superalloy: Experiments and modelling" Materials Science and Engineering: A 527:7-8 (2010) 1655-1664

[6] T. Billot, P. Villechaise, M. Jouiad, J. Mendez "Creep-fatigue behavior at high temperature of a UDIMET 720 nickel-base superalloy" International Journal of Fatigue, 32:5 (2010) 824-829

[7] P. Lin, G. Palumbo, U. Erb , K.T. Aust "Influence of grain boundary character distribution on sensitization and intergranular corrosion of alloy 600" Scripta Metallurgica et Materialia 33 (1995)1387-1392

[8] V. Thaveeprungsriporn, G.S. Was "The role of coincidence-site-lattice boundaries in Creep of Ni-16Cr-9Fe at $360^{\circ} \mathrm{C} "$ Metallurgical and Materials Transactions A 28 (1997) 2101-2112

[9] E.M. Lehockey, G. Palumbo "On the creep behaviour of grain boundary engineered nickel" Materials Science and Engineering A 237 (1997) 168-172

[10] Y. Gao, M. Kumar, R.K. Nalla, R.O. Ritchie "High cycle fatigue of a nickel-base superalloy ME3 at ambient and elevated temperature: Role of grain boundary engineering" Metallurgical and Materials Transactions A 36 (2005) 3325-3333

[11] L. Tan, K. Sridharan, T.R. Allen "The effect of grain boundary engineering on the oxidation behavior of INCOLOY alloy $800 \mathrm{H}$ in supercritical water" Journal of Nuclear Materials 348 (2006) 263-271

[12] L. Tan, K. Sridharan, T.R. Allen "Effect of thermomechanical processing on grain boundary character distribution of a Nickel-based superalloy" Journal of Nuclear Materials 371 (2007) 171-175

[13] T. Watanabe "An approach to grain boundary design for strong and ductile polycrystals", Res. Mechanica 11:1 (1984) 47-84

[14] V. Randle "Twinning-related grain boundary engineering" Acta Materialia 52 (2004) 4067-4081 
[15] J. Miao, T.M. Pollock, J. Wayne Jones "Crystallographic fatigue crack initiation in nickelbased superalloy René 88DT at elevated temperature" Acta Materialia 57:20 (2009) 5964-5974

[16] N. Souaï, N. Bozzolo, L. Nazé, Y. Chastel and R. Logé "About the possibility of grain boundary engineering via hot-working in a nickel-base superalloy" Scripta Materialia 62 (2010) p851

[17] S.A. Saltykov, Stereometric Metallography, second edition, Moscow, Metallurgizdat (1958)

[18] A.A. Guimaraes and J.J. Jonas "Recrystallization and ageing effects associated with the high temperature deformation of Waspaloy and Inconel-718" Metallurgical Transactions A 12:9 (1981) 1655-1666

[19] F.J. Humphreys, M. Hatherly "Recrystallization and Related Annealing Phenomena" Pergamon Press, Oxford (1995)

[20] J. Ciulik and E.M. Taleff "Dynamic abnormal grain growth: A new method to produce single crystals" Scripta Materialia 61:9 (2009) 895-898

[21] M. Hillert "On the theory of normal and abnormal grain growth" Acta Metallurgica 13 (1965) 227-238

[22] K. Lücke, R. Brandt, G. Abbruzzese "Normal and abnormal grain growth as transient phenomena" Interface Science 6 (1998) 67-76

[23] H. Gleiter "The formation of annealing twins" Acta Metallurgica 17 (1969) 1421-1428

[24] M.A. Meyers and C. McCowan "The formation of annealing twins: overview and new thoughts", In: C.S. Pande, D.A. Smith, A.H. King and J. Walter, Editors, Interface Migration and Control of Microstructure, Proceedings of an International Symposium held in conjunction with ASM's Metals Congress and TMS/AIME Fall Meeting, ASM, Metals Park, OH (1984), p. 99 Detroit, MI, USA, 17-21 September, 1984

[25] C.S. Pande, M.A. Imam, B.B. Rath "Study of annealing twins in FCC metals and alloys" Metallurgical Transactions A 21 (1990) 2891-2896

[26] E.E. Underwood "Quantitaive stereology", Addsion-Wesley, Menlo Park (1970)

[27] W. Wang, Y. Dai, J. Li and B. Liu "An Atomic-Level Mechanism of Annealing Twinning in Copper Observed by Molecular Dynamics Simulation" Crystal Growth \& Design, 11 (2011) 2928-2934

[28] Q. Li, J.R. Cahoon and N.L. Richards "On the calculation of annealing twin density" Scripta Materialia 55 (2006), 1155-1158 


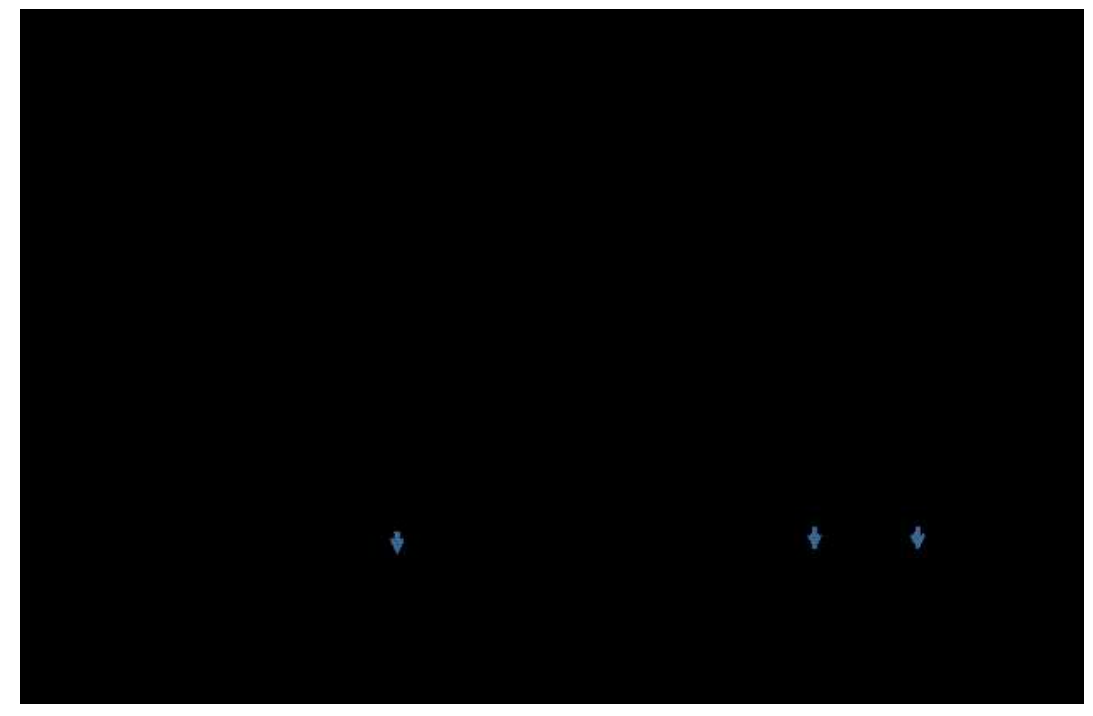

Figure 1. Synoptic diagram of the performed thermomechanical treatments

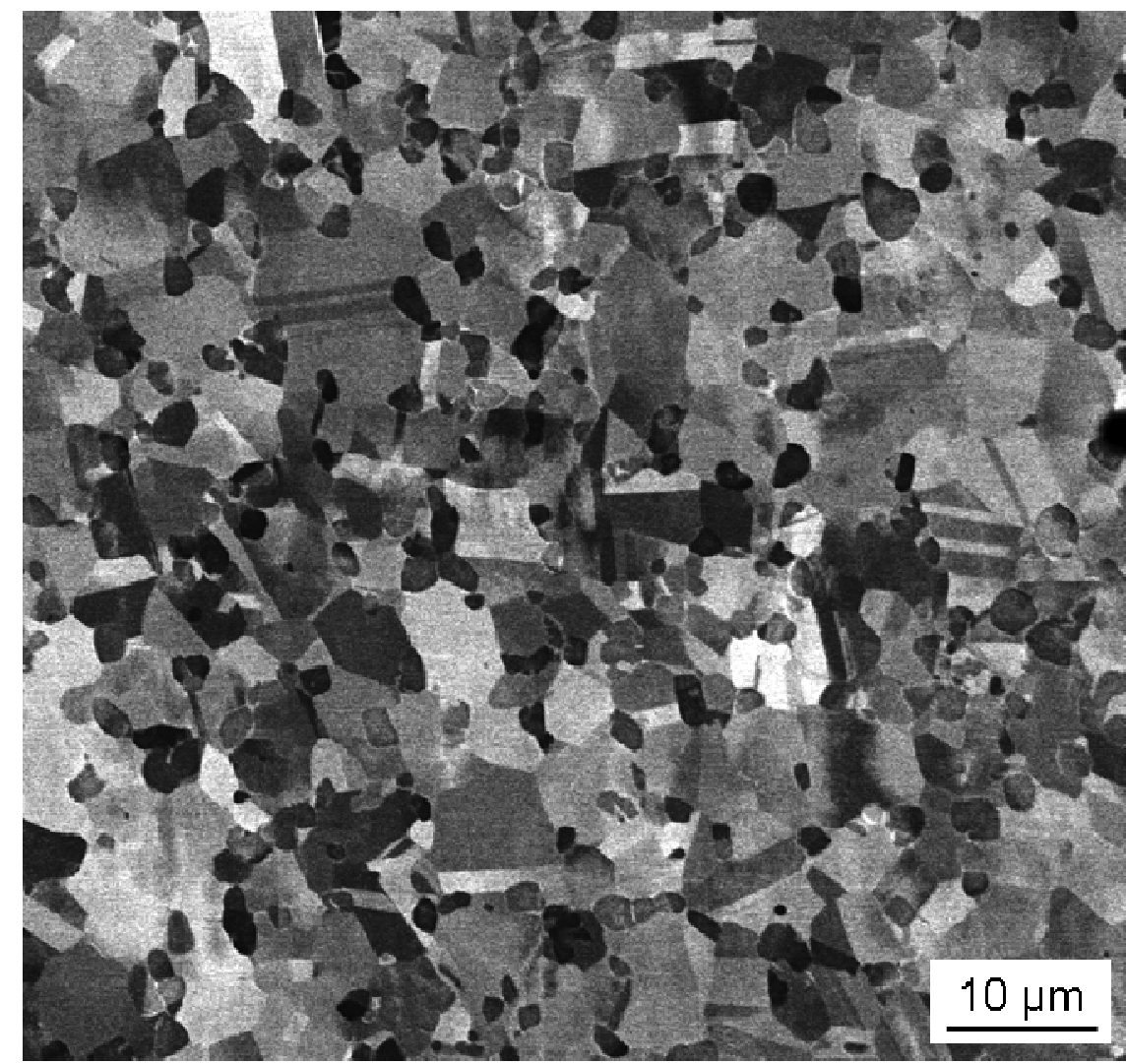

Figure 2. Back-Scattered-Electron micrograph where $\gamma^{\prime}$ precipitates are overall darker than the $y$ matrix grains due to their chemical composition which provides lower BSE intensities. (It is worth noticing that this contrast is nevertheless not suitable for phase detection by image analysis because it is in the same range as the orientation contrast within each phase.) 


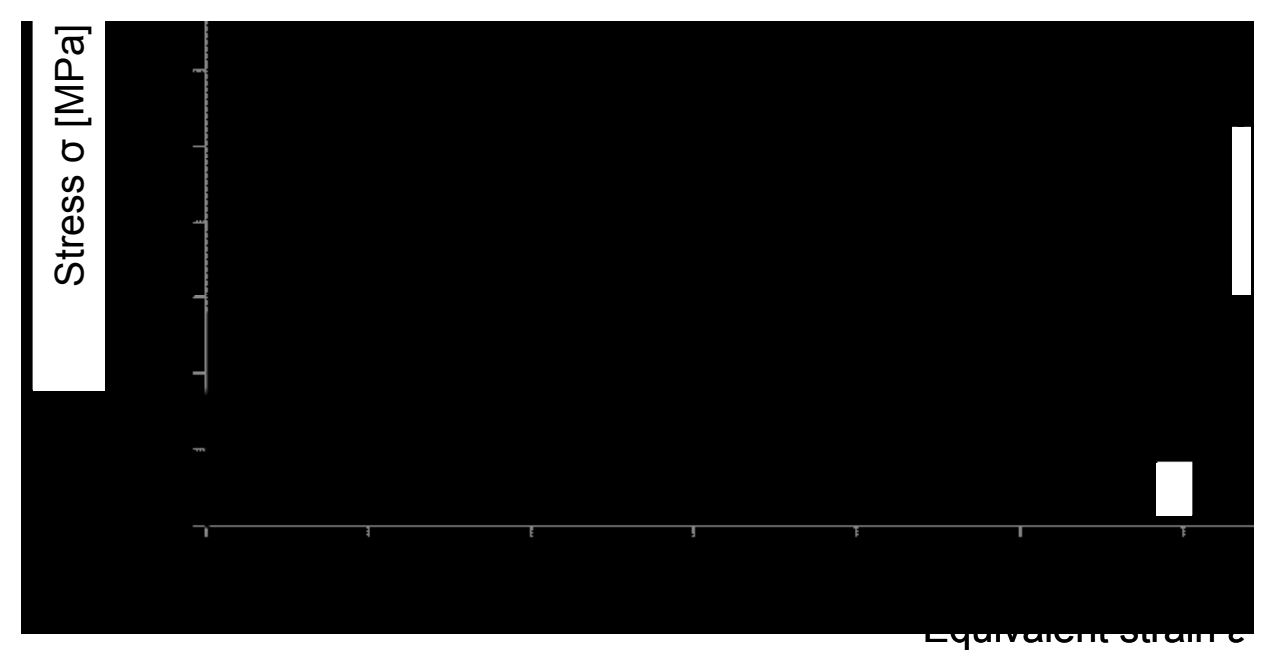

Figure 3. Stress-strain plot of the torsion rheological behavior of the PER®72 alloy. 



$50 \mu \mathrm{m}$

Figure 4. Grain structure, grain boundary network, grain size distribution histogram and average grain size for four different states, from the left column to the right: undeformed reference (annealed for 10' at $\mathrm{T}_{\text {sub }}$ and quenched), deformed to $\varepsilon=0.12$ or 0.40 or 0.63 and quenched. The extreme right bar in the grain size histograms is not a $2 \mu \mathrm{m}$ width class (like the others), but shows the area fraction of all grains with a size of at least $30 \mu \mathrm{m}$. 

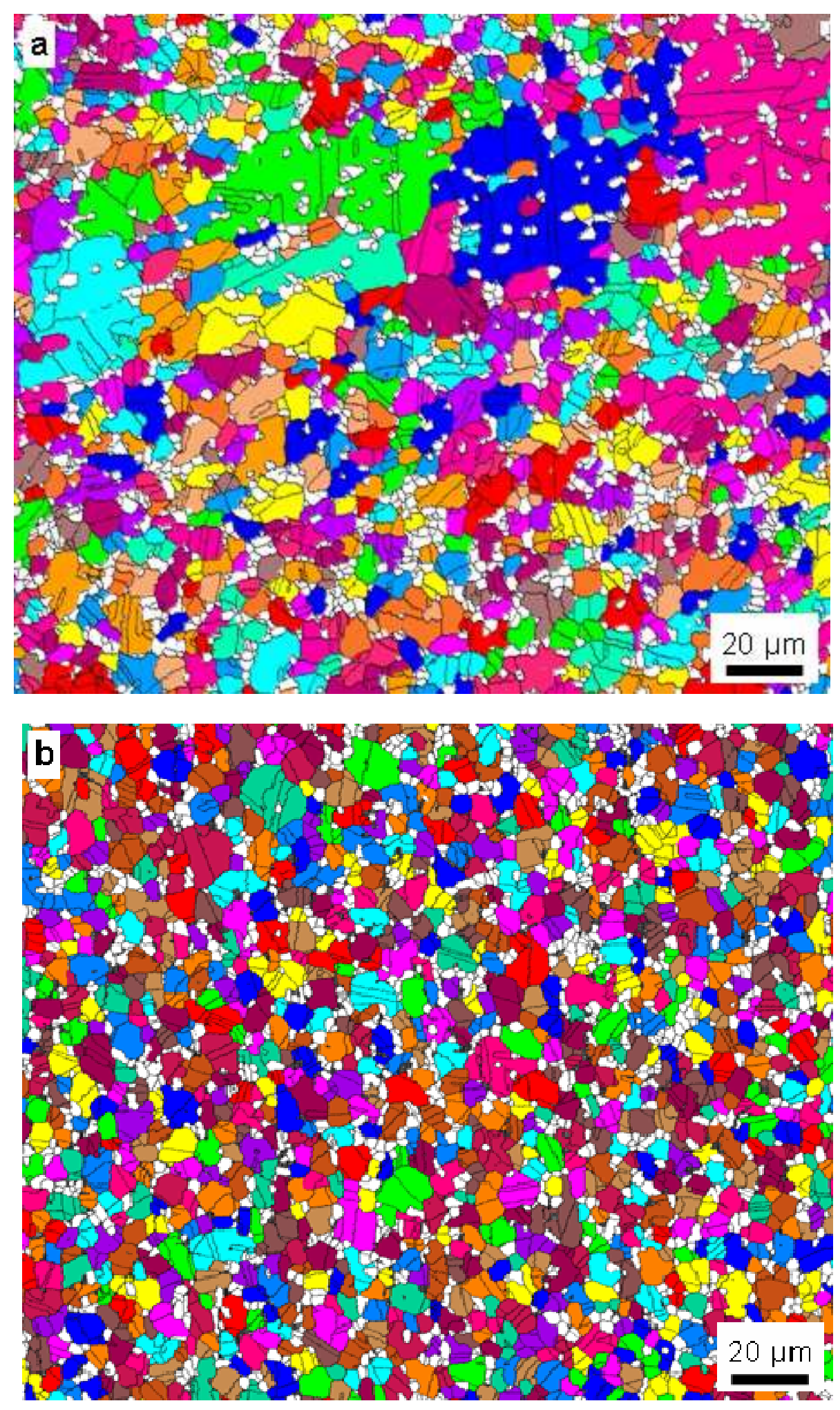

Figure 5. a) Microstructure of the sample deformed to $\varepsilon=0.12$ and quenched. b) Microstructure of the undeformed material annealed for $10^{\prime}$ at $\mathrm{T}_{\text {sub }}$ and quenched. Random grain colored EBSD maps, with all interfaces with a misorientation angle higher than $10^{\circ}$ in black. 



$50 \mu \mathrm{m}$
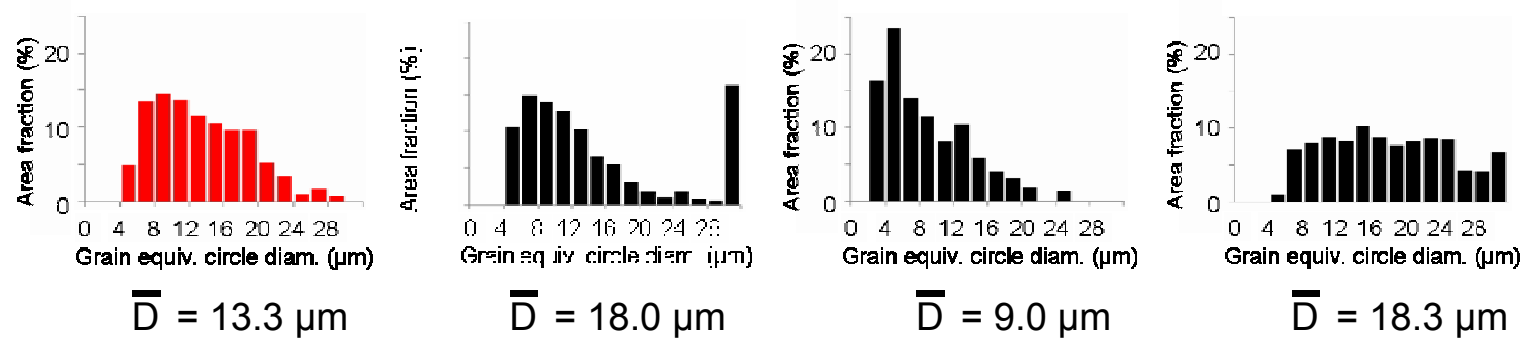

Figure 6. Grain structure, grain boundary network, grain size distribution histogram and average grain size after $30^{\prime}$ annealing at $\mathrm{T}_{\text {sub }}$ for four different states, from the left column to the right: undeformed reference, deformed to $\varepsilon=0.12$ or 0.40 or 0.63 and quenched. 

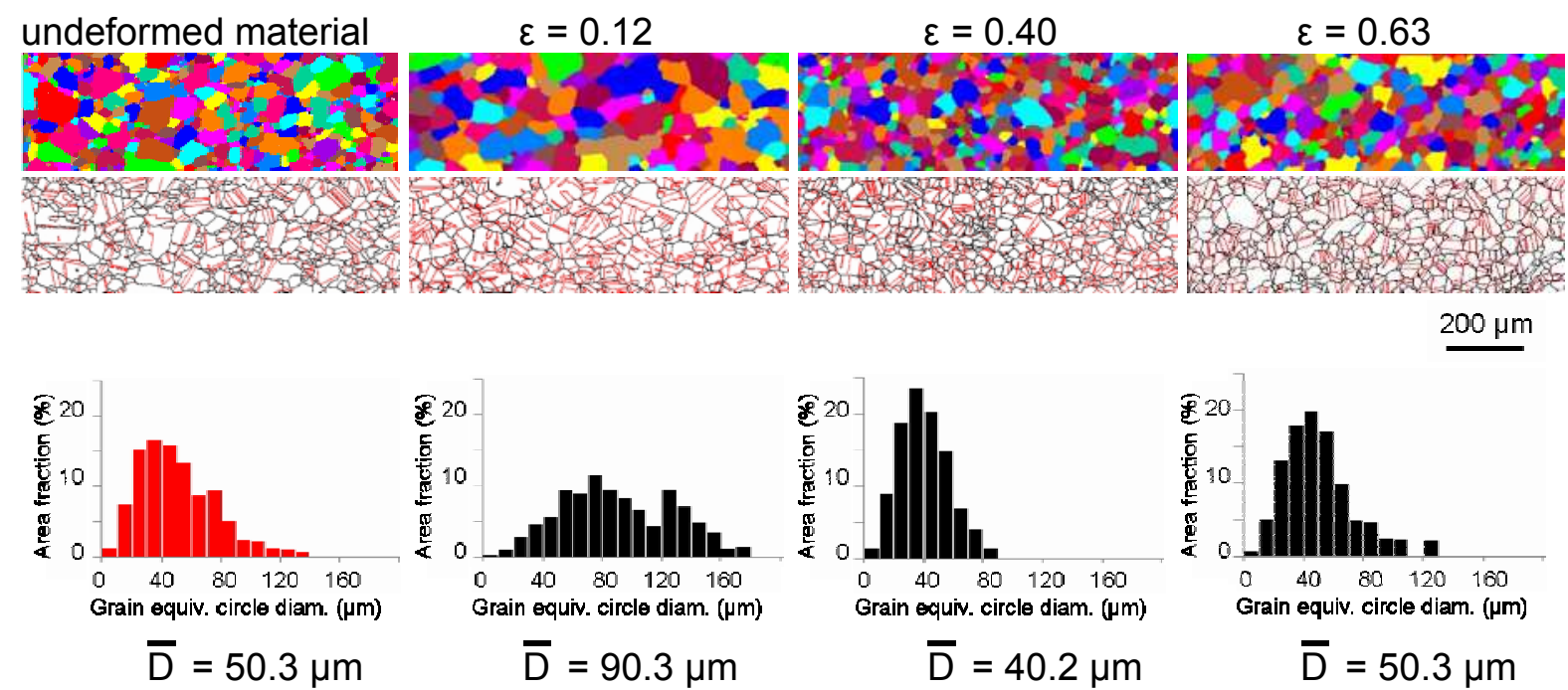

$200 \mu \mathrm{m}$

Figure 7. Grain structure, grain boundary network, grain size distribution histogram and average grain size after 30 ' annealing at $\mathrm{T}_{\text {sup }}$ for four different states, from the left column to the right: undeformed reference, deformed to $\varepsilon=0.12$ or 0.40 or 0.63 and quenched.



Figure 8. Average grain size after deformation to different strains and quenching, and followed by subsequent annealing for $30^{\prime}$ at $\mathrm{T}_{\text {sup }}$ 


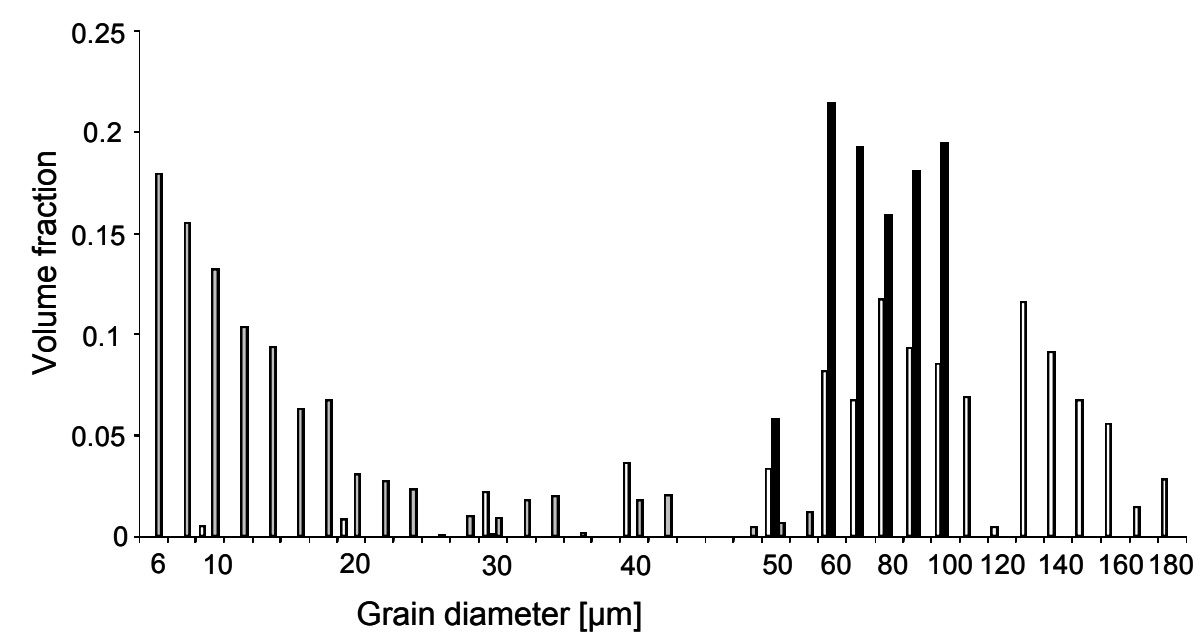

b) 0.12 prestrain

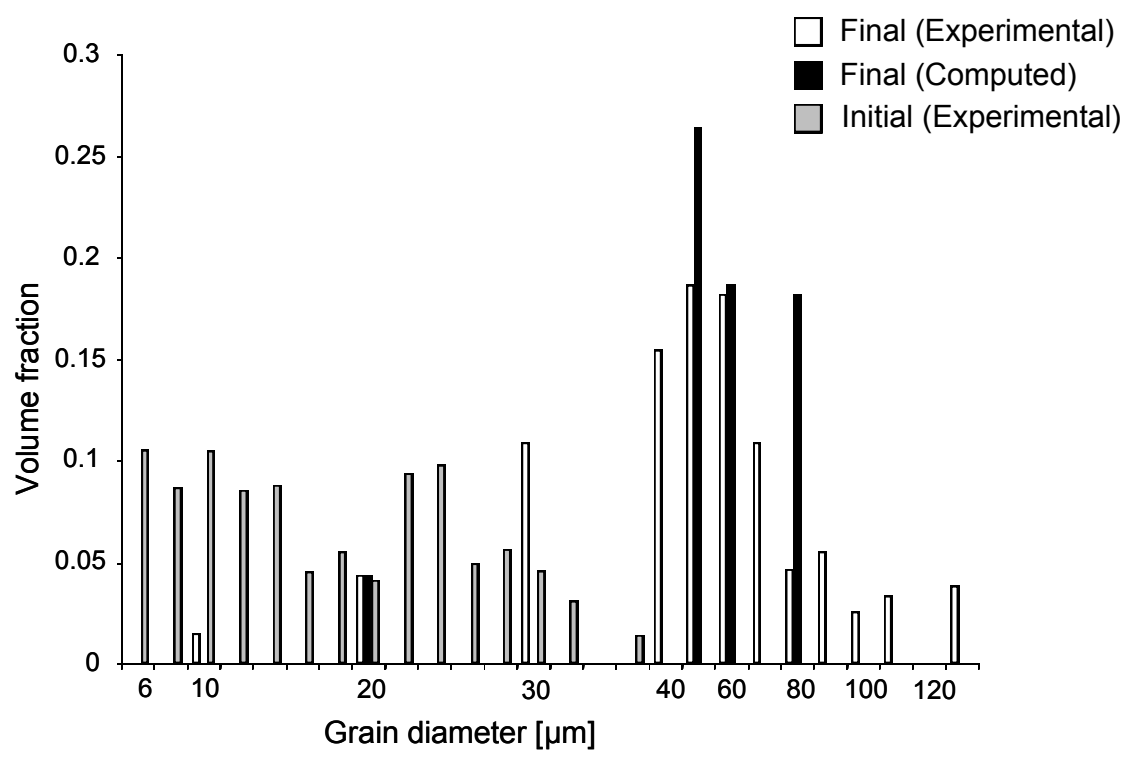

a) 0.63 prestrain

Figure 9. Evolution of 3D grain size distributions during a supersolvus heat treatment, for two samples pre-strained at a) $\varepsilon=0.63$, and b) $\varepsilon=0.12$. In order to show both initial and final distribution on the same plot, the grain size class width has been defined as $2 \mu \mathrm{m}$ for small grains ad $10 \mu \mathrm{m}$ for large ones. This is the reason for the abscise axis scale not being linear. 


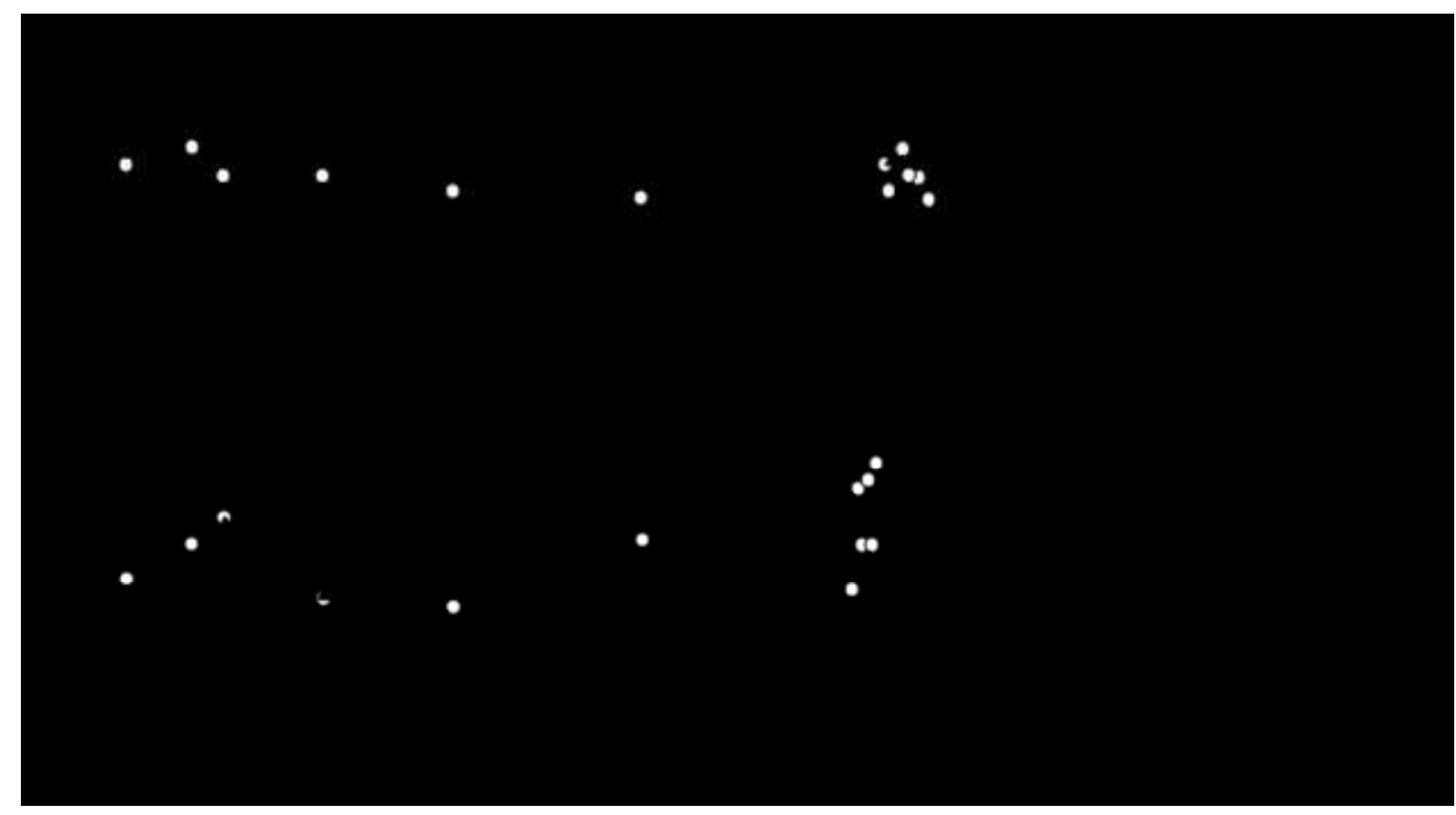

Figure 10. a,b) $\Sigma 3$ boundary density as a function of strain and average grain size. c) incoherent $\Sigma 3$ boundary proportion as function of strain. d) change in $\Sigma 3$ boundary density when reducing the misorientation angular tolerance from $4^{\circ}$ to $2^{\circ}$, as a function of the average grain size.

Quenched microstructures ( $\mathbf{(})$ and after subsequent $30^{\prime}$ annealing at $T_{\text {sub }}(\boldsymbol{\square})$ or at $T_{\text {sup }}(\mathbf{\square})$.

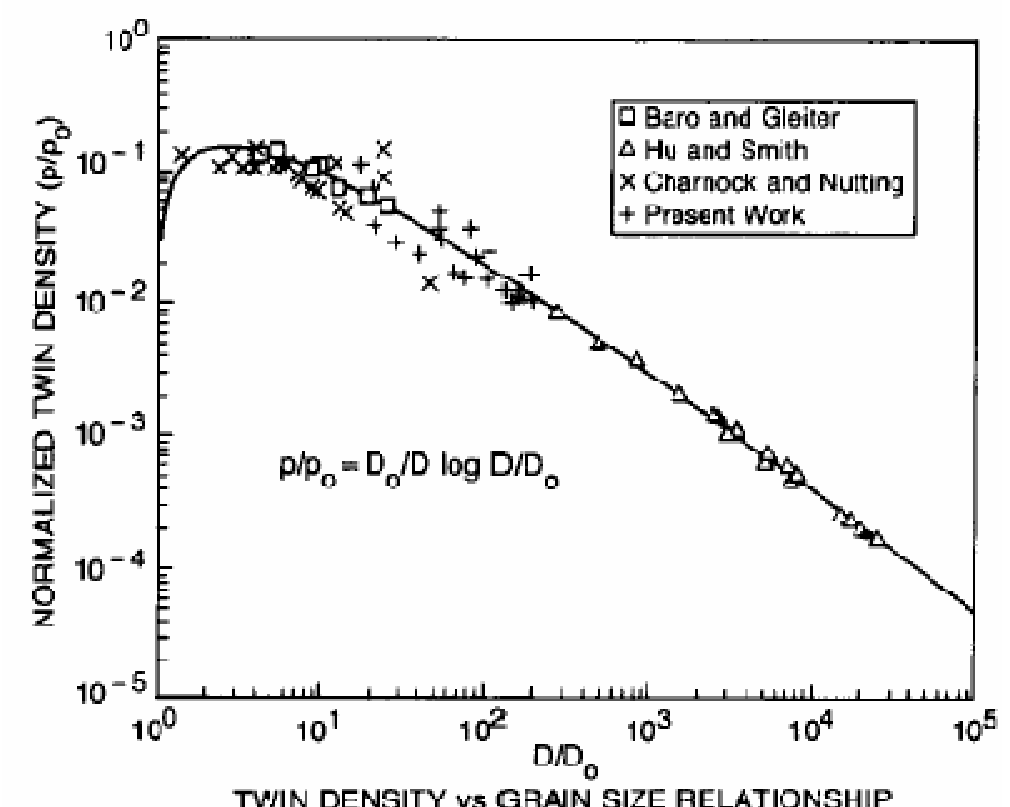

Figure 11. Relationship between twin density and grain size: experimental data and Pande's model prediction [25]. 


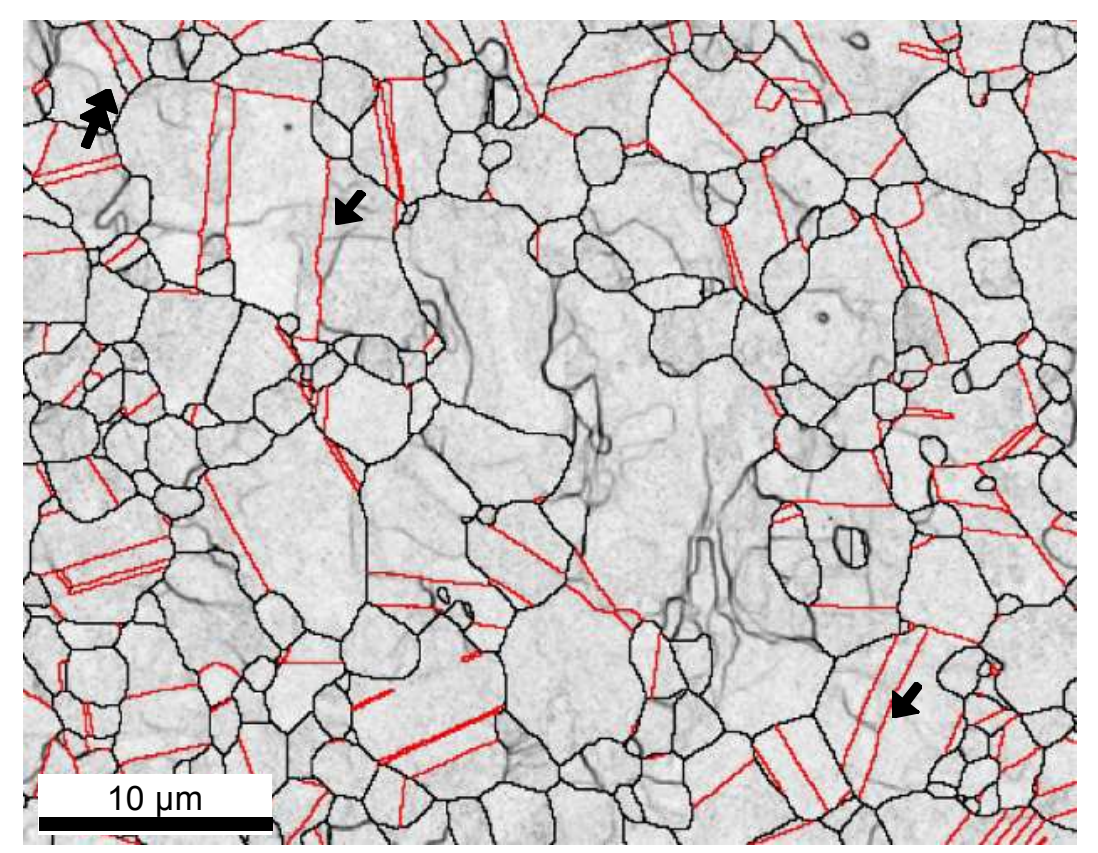
average local misorientation $\left({ }^{\circ} . \mu \mathrm{m}^{-1}\right)$

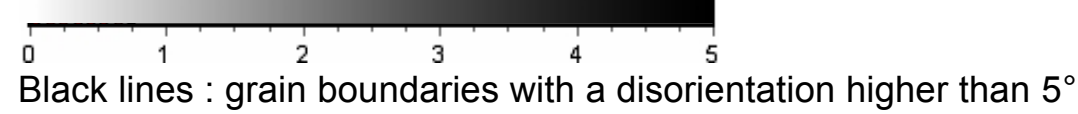

Red lines : $\Sigma 3$ boundaries ( $4^{\circ}$ tolerance)

Figure 11. High resolution EBSD map (100 nm step size) of a deformed and quenched sample $(\varepsilon=$ 0.24 at $10^{-2} \mathrm{~s}^{-1}$ ). The grayscale is related to the local orientation gradient (average misorientation angle of a pixel with all others located at $0.5 \mu \mathrm{m}$, divided by that distance). Disorientations higher than $5^{\circ}$ were ignored in the calculation of the local orientation gradients. $\Sigma 3$ boundaries $\left(4^{\circ}\right.$ tolerance with respect to the theoretical misorientation) and grain boundaries (disorientation higher than $5^{\circ}$ ) are also plotted, in red and black, respectively. 


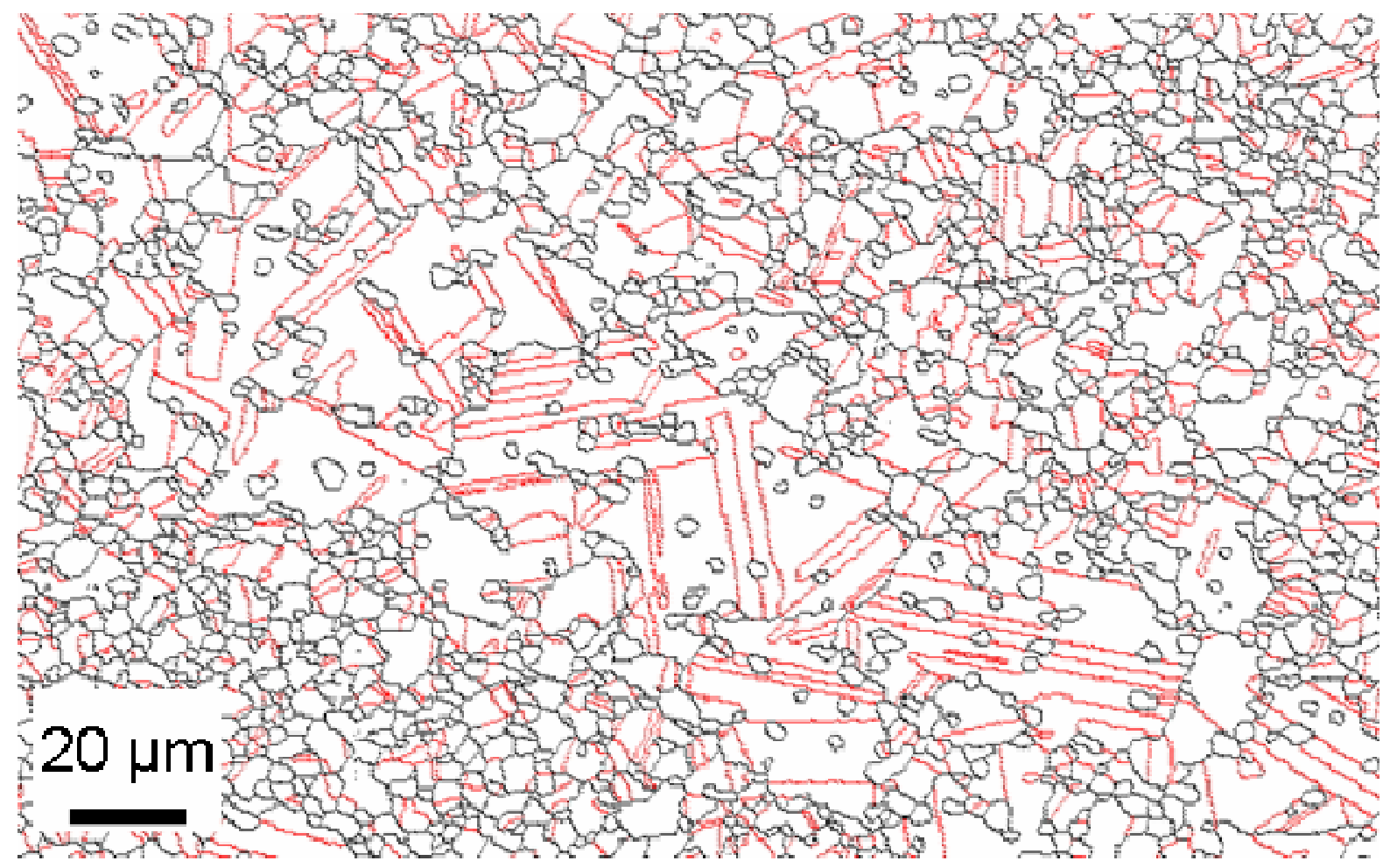

Figure 13. EBSD map of the sample annealed at the subsolvus temperature after straining to $\varepsilon=0.12$, showing grain and interphase boundaries with a disorientation higher than $10^{\circ}$ (in black), and $\Sigma 3$ twin boundaries (in red). 